Pacific

Journal of

Mathematics

GENERALIZED WRONSKIANS AND WEIERSTRASS WEIGHTS

Christopher Towse 


\title{
GENERALIZED WRONSKIANS AND WEIERSTRASS WEIGHTS
}

\author{
Christopher Towse
}

\begin{abstract}
Given a point $P$ on a smooth projective curve $C$ of genus $g$, one can determine the Weierstrass weight of that point by looking at a certain Wronskian. In practice, this computation is difficult to do for large genus. We introduce a natural generalization of the Wronskian matrix, which depends on a sequence of integers $s=m_{0}, \ldots, m_{g-1}$ and show that the determinant of our matrix is nonzero at $P$ if and only if $s$ is the non-gap sequence at $P$.

As an application, we compute the weights of certain points on the $F_{9}$ and $F_{10}$, the 9 th and 10 th Fermat curves. These weights correspond to the expected weights predicted in an earlier paper.
\end{abstract}

\section{Introduction.}

One of the fundamental facts about Weierstrass points (and generalizations of Weierstrass points) is that any given curve has only a finite number of them; that the total Weierstrass weight of all the points on a curve is finite. Thus, one of the basic problems in the study of Weierstrass points is, once found, to count the Weierstrass weight of those points. One would at least like to know if one has found all of the Weierstrass points on one's curve of interest.

Fermat curves $F_{n}: X^{n}+Y^{n}+Z^{n}=0$ are of particular interest since they have so many automorphisms, and thus (by Lewittes' Theorem $[\mathbf{L}]$ ) so many known Weierstrass points. Hasse $[\mathbf{H a}]$ showed that the weight of a point with $X Y Z=0$ is $(n-1)(n-2)(n-3)(n+4) / 24$ by demonstrating the existence of certain holomorphic differentials. In $[\mathbf{T}]$, we exploited the simplicity of order two automorphisms to get a lower bound on the Weierstrass weight of a second class of well-known Weierstrass points on Fermat curves. Further, we showed, with the aid a computer, that the lower bound corresponded to the actual weights for $n \leq 8$. For $n=8$, this was a new result. It seemed unlikely to be able to repeat this for higher degree (more relevantly, higher genus) Fermat curves. Below, however, we do demonstrate that the lower bound given in $[\mathbf{T}]$ is exact, for $n=9$ and 10 . 


\section{Preliminaries.}

Throughout, we will consider monotonically increasing sequences of nonnegative integers $s=\left(m_{0}, \ldots, m_{g-1}\right)$. We define the weight of such a sequence to be $\operatorname{wt}(s)=\operatorname{wt}\left(m_{0}, \ldots, m_{g-1}\right)=\sum_{i=0}^{g-1}\left(m_{i}-i\right)$.

The idea of the weight is to measure how much a given sequence differs from a "standard" sequence. In this case, we are merely comparing $s$ to $s_{0}=(0,1, \ldots, g-1)$.

Let $C$ be a smooth, projective curve of genus $g$ defined over $K=\mathbb{C}$ or any other algebraically closed, characteristic zero field. Let $P$ be a point on $C$.

Definition. Given any $k$-dimensional $K$-vector space, $A$, of holomorphic differentials on $C$, we say a basis for $A, \omega_{0}, \ldots, \omega_{k-1}$, is adapted to $P$ if $0 \leq \operatorname{ord}_{P} \omega_{0}<\operatorname{ord}_{P} \omega_{1}<\cdots<\operatorname{ord}_{P} \omega_{k-1}$.

It is well-known (see $[\mathbf{F}-\mathbf{K}]$ ) that any such $A$ has a basis adapted to $P$, for any $P \in C$. It is easy to see that the numbers $\operatorname{ord}_{P} \omega_{i}$ do not depend on the choice of basis, as long as the basis is adapted for $P$.

Definition. If we let $A$ be the space of all holomorphic differentials on $C$, and we let $n_{i}=\operatorname{ord}_{P} \omega_{i}$, then the sequence of (monotonically increasing, nonnegative) integers $n_{0}, \ldots, n_{g-1}$ is called the nongap sequence of $P$.

Definition. We define the (Weierstrass) weight of $P$ to be the weight of the nongap sequence at $P$.

Note that this differs from the "classical" nongap sequence defined using orders of poles of functions at $P$. However, the ideas are related via the Riemann-Roch Theorem, and the Weierstrass weight of a point is the same, using either approach.

\section{Main Results.}

Let $\left\{\omega_{0}, \ldots, \omega_{g-1}\right\}$ be a basis of holomorphic differentials on $C$. Let $x$ be a local uniformizing parameter on some Zariski open set of $C$ containing $P$. Then we can write each differential as $\omega_{j}=f_{j} d x$ for some function $f_{j}$. Let $\mathcal{F}=\left\{f_{0}, \ldots, f_{g-1}\right\}$.

A fundamental fact (due to Hurwitz $[\mathbf{H u}]$ ) is that the weight of a point $P$ is equal to the order of vanishing at $P$ of the determinant of the Wronskian matrix whose first row is $\mathcal{F}$. First, we relate the weight of $P$ to the weights of various sequences $s$.

Definition. Suppose we have chosen any fixed set of functions $\Phi=$ $\left\{\phi_{j}(x)\right\}_{j=0}^{k-1}$. We define $M_{\Phi}\left[m_{0}, \ldots, m_{k-1}\right]=M\left[m_{0}, \ldots, m_{k-1}\right]=M[s]$ to be the matrix whose $i$ th row $(i=0, \ldots k-1)$ is $\left(\phi_{0}^{\left(m_{i}\right)}, \ldots, \phi_{k-1}^{\left(m_{i}\right)}\right)$ where $\phi^{(m)}$ denotes the $m$ th derivative with respect to $x$. 
So $M[0, \ldots, k-1]$ is a Wronskian matrix with first row $\left[\phi_{0}, \ldots, \phi_{k-1}\right]$. We will abbreviate this as $W_{\Phi}$.

Proposition 1. The dth derivative of the Wronskian determinant is equal to a sum of determinants of matrices $M[s]$ where $s$ has weight $d$.

Proof. We use induction on $d$. For the case $d=0$, we note that the only sequence of weight zero is $s_{0}=(0,1, \ldots, k-1)$. As noted above, $M\left[s_{0}\right]$ is the Wronskian itself.

For general $d$, we look at the derivative of the determinant of one of the matrices $M[s]$. Let $D$ denote differentiation with respect to $x$. Using linearity of the determinant in the rows of $\mathrm{M}$, we see that

$$
\begin{aligned}
D\left(\operatorname{det} M\left[m_{0}, \ldots, m_{k-1}\right]\right)=\operatorname{det} M\left[m_{0}+\right. & \left.1, m_{1}, \ldots, m_{k-1}\right]+\cdots \\
& +\operatorname{det} M\left[m_{0}, m_{1}, \ldots, m_{k-1}+1\right] .
\end{aligned}
$$

If the old sequence $\left(m_{0}, \ldots, m_{k-1}\right)$ has weight $d$, then any of the new sequences $\left(m_{0}, \ldots, m_{i}+1, \ldots, m_{k-1}\right)$ has weight $d+1$.

It should be noted that not all of such sequences will be strictly monotonic, but for any of those sequences, $s, M[s]$ will have a repeated row, and will therefore have determinant identically zero. Note also that the same $M[s]$ may appear more than once in the sum. For instance, with $g=4$ and $k=3$, we get

$$
D^{(3)} \operatorname{det} W=\operatorname{det} M[0,2,3,4]+2 \operatorname{det} M[0,1,3,5]+\operatorname{det} M[0,1,2,6] .
$$

The point of the proposition is the observation that the sequences appearing in our $M[s]$ notation are related to the weight of a point. The following theorem elaborates on this connection.

Theorem 2. Let $s=\left(m_{0}, \ldots, m_{g-1}\right)$ be a monotonically increasing sequence of nonnegative integers. Let $P$ be a point of $C$ and let $\mathcal{F}$ be as above. Suppose $\operatorname{wt}(s) \leq \operatorname{wt}(P)$. Then $\operatorname{det} M_{\mathcal{F}}[s](P) \neq 0$ if and only if $s$ is the nongap sequence of $P$.

That is, the determinant of the matrix $M=M\left[m_{0}, \ldots, m_{g-1}\right]$, evaluated at the point $P$, is zero for all sequences of weight less than or equal to the weight at $P$, except for the actual nongap sequence of $P$.

Proof. It is clear that changing the basis $\mathcal{F}$ will only change the determinant by a nonzero multiple. So we may assume that $\omega_{0}, \ldots, \omega_{g-1}$ is a basis for the space of holomorphic differentials of $C$ adapted to $P$. Let $\left(n_{0}, \ldots, n_{g-1}\right)$ be the nongap sequence at $P$.

Since $x$ is a local uniformizing parameter at $P, P$ is not in the support of the divisor $(d x)$. In other words, $\operatorname{ord}_{P} d x=0$. Thus, $\operatorname{ord}_{P} f_{i}=n_{i}$. So $D^{(k)}\left(f_{i}\right)(P)=0$ for $k<n_{i}$ and $D^{\left(n_{i}\right)}\left(f_{i}\right)(P) \neq 0$. 
In particular, if we consider the case when $m_{i}=n_{i}$ for all $i$, we see that $M$ is lower triangular, with nonzero entries on the diagonal. Thus, $\operatorname{det} M \neq 0$.

Now, suppose $m_{i} \neq n_{i}$ for some $i$. Since $\operatorname{wt}\left(m_{0}, \ldots, m_{g-1}\right) \leq \operatorname{wt}\left(n_{0}, \ldots\right.$, $\left.n_{g-1}\right)$, we know there is some $m_{i}<n_{i}$. Let $I$ be the smallest index with $m_{I}<n_{I}$. Then the first $I$ rows of $M$ can only have nonzero entries in (at most) the first $I-1$ places. They are all contained in the $(I-1)$-dimensional $K$-vector space $K^{I-1} \times\{0\}^{g-I+1}$. Thus, the first $I$ rows of $M$ are linearly dependent. We conclude that $\operatorname{det} M=0$.

Let $\Phi=\left\{\phi_{0}, \ldots, \phi_{k-1}\right\}$. As above, $W_{\Phi}$ is the corresponding Wronskian matrix. A basic property of Wronskians is this: If $\mathcal{G}=\left\{h \phi_{0}, \ldots, h \phi_{k-1}\right\}$ then

$$
\operatorname{det} W_{\mathcal{G}}=h^{k} \operatorname{det} W_{\Phi}
$$

This equality is not true if we replace the Wronskians by general matrices of the form $M[s]$. However, we do have the following result.

Proposition 3. Let $\mathcal{F}$ be a set of functions $\left\{f_{1}, \ldots, f_{g}\right\}$ so that the differentials $\omega_{i}=f_{i} d x$ form a basis for the space of holomorphic differentials of $C$. Let $h=h(x)$ be another function and let $\mathcal{G}=\left\{h f_{0}, \ldots, h f_{g-1}\right\}$. Let $n_{0}, \ldots, n_{g-1}$ be the nongap sequence at $P$. Let $s=\left(m_{0}, \ldots, m_{g-1}\right)$ be a sequence with $m_{i} \leq n_{i}$ for all $i$. Then

$$
\operatorname{det} M_{\mathcal{G}}[s](P)=h^{g} \operatorname{det} M_{\mathcal{F}}[s](P) .
$$

Proof. The $i$ th row of $M_{\mathcal{G}}$ is

$$
\begin{gathered}
{\left[\begin{array}{lll}
\left(h f_{0}\right)^{\left(m_{i}\right)} & \ldots & \left(h f_{g-1}\right)^{\left(m_{i}\right)}
\end{array}\right]} \\
=\left[\begin{array}{ccc}
\sum_{r=0}^{m_{i}}\left(\begin{array}{c}
r \\
m_{i}
\end{array}\right) h^{(r)} f_{0}^{\left(m_{i}-r\right)} & \ldots & \sum_{r=0}^{m_{i}}\left(\begin{array}{c}
r \\
m_{i}
\end{array}\right) h^{(r)} f_{g-1}^{\left(m_{i}-r\right)}
\end{array}\right] .
\end{gathered}
$$

Expanding the determinant of $M_{\mathcal{G}}[s]$ by linearity in the rows, we get a sum of determinants of matrices with $i$ th row equal to

$$
\left[\begin{array}{lll}
h^{(r)} f_{0}^{\left(m_{i}-r\right)} & \cdots & h^{(r)} f_{g-1}^{\left(m_{i}-r\right)}
\end{array}\right]
$$

where $0 \leq r \leq m_{i}$. We can pull out $h^{(r)}$ from these rows.

Let us consider one of the matrices whose determinant is in our sum. Its $i$ th row is of the form

$$
\left[\begin{array}{lll}
f_{0}^{\left(t_{i}\right)} & \cdots & f_{g-1}^{\left(t_{i}\right)}
\end{array}\right]
$$

where $t_{i} \leq m_{i} \leq n_{i}$. So this matrix is just $M_{\mathcal{F}}[\tilde{s}]$, where $\tilde{s}=\left(t_{0}, \ldots, t_{g-1}\right)$. Plugging in the point $P$, we know that $\operatorname{det} M_{\mathcal{F}}[\tilde{s}](P)=0$ unless $t_{i}=n_{i}$ for all $i$, by the theorem.

We have two cases. First, if $m_{i}<n_{i}$ for some $i$, then we see that all the determinants in our sum vanish at $P$, by the theorem. And we see that $\operatorname{det} M_{\mathcal{F}}[s](P)=0$ by the theorem, as well. 
If, on the other hand, $s$ was the nongap sequence of $P$ to begin with, then there is exactly one nonvanishing term in our sum. It is the one with $\tilde{s}=s$. That is, $r=0$ in all cases. So we have factored out $h^{(0)}=h$ from each of the $g$ rows. Also, the factors $\left(\begin{array}{c}r \\ m_{i}\end{array}\right)=\left(\begin{array}{c}0 \\ m_{i}\end{array}\right)$ are all 1. We get $\operatorname{det} M_{\mathcal{G}}[s](P)=h^{g} \operatorname{det} M_{\mathcal{F}}[s](P)$.

\section{Examples - Fermat curves.}

Consider the $n$th Fermat curve $F_{n}: X^{n}+Y^{n}+Z^{n}=0$. Dehomogenized at $Z \neq 0$, this is given by $x^{n}+y^{n}+1=0$.

Definition. Any point $P \in F_{n}$ with $X Y Z=0$ will be called a trivial Weierstrass point.

Let $\zeta_{n}$ be a primitive $n$th root of unity. Consider the involutions, $T$, of $F_{n}$ given by

$$
[X, Y, Z] \mapsto\left[\zeta_{n}^{j} Y, \zeta_{n}^{-j} X, Z\right], \quad j=0, \ldots, n-1 .
$$

We could also have $T$ switch $X$ and $Z$ or $Y$ and $Z$, of course, for a total of $3 n$ involutions.

Definition. Any Weierstrass point $P \in F_{n}$ fixed by one of the involutions $T$ will be called a diagonal Weierstrass point.

We should note that for odd $n$ there are diagonal Weierstrass points which are also trivial Weierstrass points. Since the trivial points are well understood (again, see $[\mathbf{H a}]$ ), we will ignore them in the following discussion.

We showed in $[\mathbf{T}]$ that any diagonal point, $P$, has exactly $q$ odd nongaps and $g-q$ even ones. Here

$$
q= \begin{cases}(n-1)(n-3) / 4 & n \text { odd } \\ (n-2)^{2} / 4 & n \text { even }\end{cases}
$$

is the genus of the any of the quotient curves $F_{n} /\langle T\rangle$.

Let

$$
s=(0,1, \ldots, 2 q-1,2 q, 2 q+2, \ldots, 2(g-q-1)) .
$$

The weight of $s$ is the so-called expected weight of $P, \mathrm{wt}_{e}(P)$. We know from $[\mathbf{T}]$ that

$$
\operatorname{wt}(P) \geq \operatorname{wt}_{e}(P)= \begin{cases}(n-1)(n-3) / 8 & n \text { odd } \\ (n-2)(n-4) / 8 & n \text { even. }\end{cases}
$$

Proposition 4. Let

$$
\mathcal{H}=\left\{x^{i} y^{j}: 0 \leq i \leq n-3-j, 1 \leq j \leq n-3\right\},
$$

$P=\left((-1 / 2)^{(1 / n)},(-1 / 2)^{(1 / n)}\right)$, and $s^{\prime}=(n-2, n-1, \ldots, 2 q-1,2 q, 2 q+$ $2, \ldots, 2(g-q-1))$. Then $\operatorname{det} M_{\mathcal{H}}\left[s^{\prime}\right](P) \neq 0$ if and only if the weight of $P$ is equal to the expected weight, $\mathrm{wt}_{e}(P)$, given above. 
Proof. It is easy to check that

$$
\left\{x^{i} / y^{j} d x: \quad 0 \leq i \leq j-2 \text { and } j=2, \ldots, n-1\right\}
$$

is a basis for the space of holomorphic differentials on $F_{n}$. So we may let $\mathcal{F}$ be the corresponding set of functions of the form $x^{i} / y^{j}$. Let $h=y^{n-1}$. Then $\mathcal{G}=\left\{x^{i} y^{j}: 0 \leq i \leq n-3-j, 0 \leq j \leq n-3\right\}$. Proposition 3 says that $\operatorname{det} M_{\mathcal{G}}[s](P)=h^{g} \operatorname{det} M_{\mathcal{F}}[s](P)$ for any sequence $s$ of weight less than or equal to the weight of $P$.

Further, since the first $n-2$ functions of $\mathcal{G}$ are $\left\{1, x, \ldots, x^{n-3}\right\}$, we see that

$$
y^{-g(n-1)} \operatorname{det} M_{\mathcal{F}}[s](P)=\operatorname{det} M_{\mathcal{G}}[s](P)=\alpha \operatorname{det} M_{\mathcal{H}}\left[s^{\prime}\right](P)
$$

where $\alpha=\prod_{i=0}^{n-2} i$ !. Since $\operatorname{det} M_{\mathcal{F}}[s](P)$ is nonzero if and only if $\operatorname{wt}(P)=$ $\operatorname{wt}(s)$, by the theorem and since $\operatorname{ord}_{P} h=0$, we are done.

Proposition 5. The nontrivial diagonal Weierstrass points on $F_{9}$ have weight 6.

Proof. Using the set of functions $\mathcal{H}$ from the corollary, we had Mathematica compute the matrix $M=M_{\mathcal{H}}[7, \ldots, 24,26,28,30](P)$.

Factoring out as much as we could from the rows and columns of the matrix, we were able to have Mathematica compute the remaining determinant:

$\operatorname{det} M=(-1)^{2 / 3}(2)^{2 / 9} 2^{438} 3^{129} 5^{67} 7^{44} 11^{25} 13^{18} 17^{23} 19^{9} 23^{6} 29^{2}(53)(967)(2141) \kappa$ where $\kappa$ is a composite integer on the order of $10^{85}$ with no small factors:

(1) $\quad \kappa=4972125503975388123549399196928230101413$

$$
365278909345657357099864972271058186013692161 .
$$

This is nonzero and hence we conclude that the Weierstrass weight of the 243 conjugates of $P$ (including itself, but excluding the points with $X Y Z=0)$ are all 6 , as "expected." Furthermore, we have shown that the nongap sequence at all of these points is $(0,1, \ldots, 24,26,28,30)$.

We can do a similar computation for $F_{10}$. The genus is 36 , and the expected gap sequence is $0,1, \ldots, 31,32,34,36,38$.

Proposition 6. The diagonal Weierstrass points on $F_{10}$ have weight 6 .

Proof. Using Mathematica, we compute (with the appropriate set of functions $\mathcal{H})$ the matrix $M=M_{\mathcal{H}}[8,9, \ldots, 31,32,34,36,38](P)$. Rather than attempting to compute the exact determinant, we factor out powers of $2^{1 / 10}$ and powers of $(-1)^{1 / 10}$. This leaves a matrix with integer entries. In order to show that the determinant of this matrix is nonzero, we need only show that it is nonzero modulo $p$ for some integer $p$. First, we reduce the entries of the matrix modulo $p$ and obtain a matrix of residues. Next, we can we 
can easily find the exact determinant of this matrix. Lastly, we reduce this determinant modulo $p$ again.

In this case, the determinant is zero modulo $p$ for all primes less than 43 . Of course this only means that these primes divide the determinant. The prime 43 is the smallest which does not divide the determinant. Modulo 43, we get a nonzero determinant. (We got 17 modulo 43.) This shows that the actual nongap sequence is the one we "expected." And thus, the weight of each of the 300 points on $F_{10}$ conjugate to $\left((-1 / 2)^{1 / 10},(-1 / 2)^{1 / 10}\right)$, including itself, is 6 .

It is interesting to note that the primes which divide the determinant of $M$, in this case, include (but are not limited to) all primes less than 43 , as well as 59, 79, and 997. No other primes less than 3571 (the 500th prime) divide the determinant. (Compare with the determinant of $M$ in Proposition 5.)

In general, one should be able to check whether diagonal points have the minimal possible nongap sequence by calculating $\operatorname{det} M_{\mathcal{H}}[s](P)$.

The difficulty is in computing the higher derivatives in order to create the matrix $M$. Further, one cannot numerically calculate the determinant of $M$ without running into round-off error problems. For $n=9$, we computed this determinant exactly. This seems too difficult and slow a computation for larger $n$, at this time. For $n=10$, we reduced modulo some small primes. This works as long as the determinant is, in fact, nonzero. It is interesting, however, that the evidence does seem to suggest that the expected gap sequences are the actual ones, in general.

Also, it is intriguing that the primes $p$ for which the diagonal points coalesce with other points on the reduction of $F_{n}$ modulo $p$ (that is, the primes which divide $\operatorname{det} M$ ) seem to include most small primes as well as just a few scattered large primes.

\section{References}

[A] R. Accola, Topics in the theory of Riemann surfaces, 1595, Springer-Verlag, 1994, Lecture Notes in Mathematics.

[F-K] H. Farkus and I. Kra, Riemann Surfaces, Springer-Verlag, 1980.

[Ha] H. Hasse, Über den algebraischen Funktionenkörper der Fermatschen Gleichung, in 'Mathematische Abhandlungen', Walter de Gruyter, 1975; originally in Acta Univ. Szeged Sect. Math., 13 (1950), 195-207.

[Hu] A. Hurwitz, Ueber algebraische Gebilde mit eindeutigen Transformationen in sich, Mathematiche Annalen, 41 (1893), 403-442.

[L] J. Lewittes, Automorphisms of compact Riemann surfaces, Amer. J. Math., 85 (1963), 732-752. 
[T] C. Towse, Weierstrass weights of fixed points of an involution, Math. Proc. Camb. Phil. Soc., 122(3) (1997), 385-392.

Received August 19, 1998 and revised March 23, 1999.

Department of Mathematics and Statistics

Pomona College

Claremont, CA 91711-6348

E-mail address: ctowse@pomona.edu 